\title{
“Duplex Ureter": ¿Are we Aware of this Uncommon Finding?
}

Carlos Leal, MD, PhD*

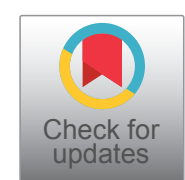

Division of Gynecologic Oncology, Department of Obstetrics and Gynecology, Universidad Autónoma de Chihuahua, Mexico

A 27-year-old, gravida 0 para 0 , female presented herself for an evaluation of recurrent urinary tract infections and severe dysmenorrhea. The ultrasound revealed a bilateral small but complex adnexal mass, suggesting endometriosis (Figure 1). Ca 125 was $88 \mathrm{U}$. Laparoscopic surgery was performed, showing evidence of infiltrative endometriosis and entrapment of the right ureter on the right pelvic sidewall (Figure 2); During the initial anatomical inspection a duplicated ureter was diagnosed (Figure 3). The laparoscopic surgery was performed to liberate both ureters and resection of the deep endometriosis without any complicated issues (Figure 4).

Congenital anomalies of the kidney and the urinary tract (CAKUT) constitute approximately 20 to 30 percent of all anomalies identified in the prenatal period. Defects can be bilateral or unilateral, and different defects often coexist in an individual patient [1].

Complete or partial duplication of the renal collecting system also referred to as a duplicated system, is the most common congenital anomaly of the urinary tract [2]. Autopsy studies report an estimated incidence of 0.8 to 5.0 percent [3]. Double collecting systems are thought to result from duplication of the ureteric bud, with the superior bud associated with the upper renal pole, and the inferior bud with the lower renal pole.

In complete duplication, the kidney has two separate pelvicalyceal systems and two ureters. The ureter from the lower collecting system usually enters the bladder in the trigone, whereas the ureter from the upper collecting system

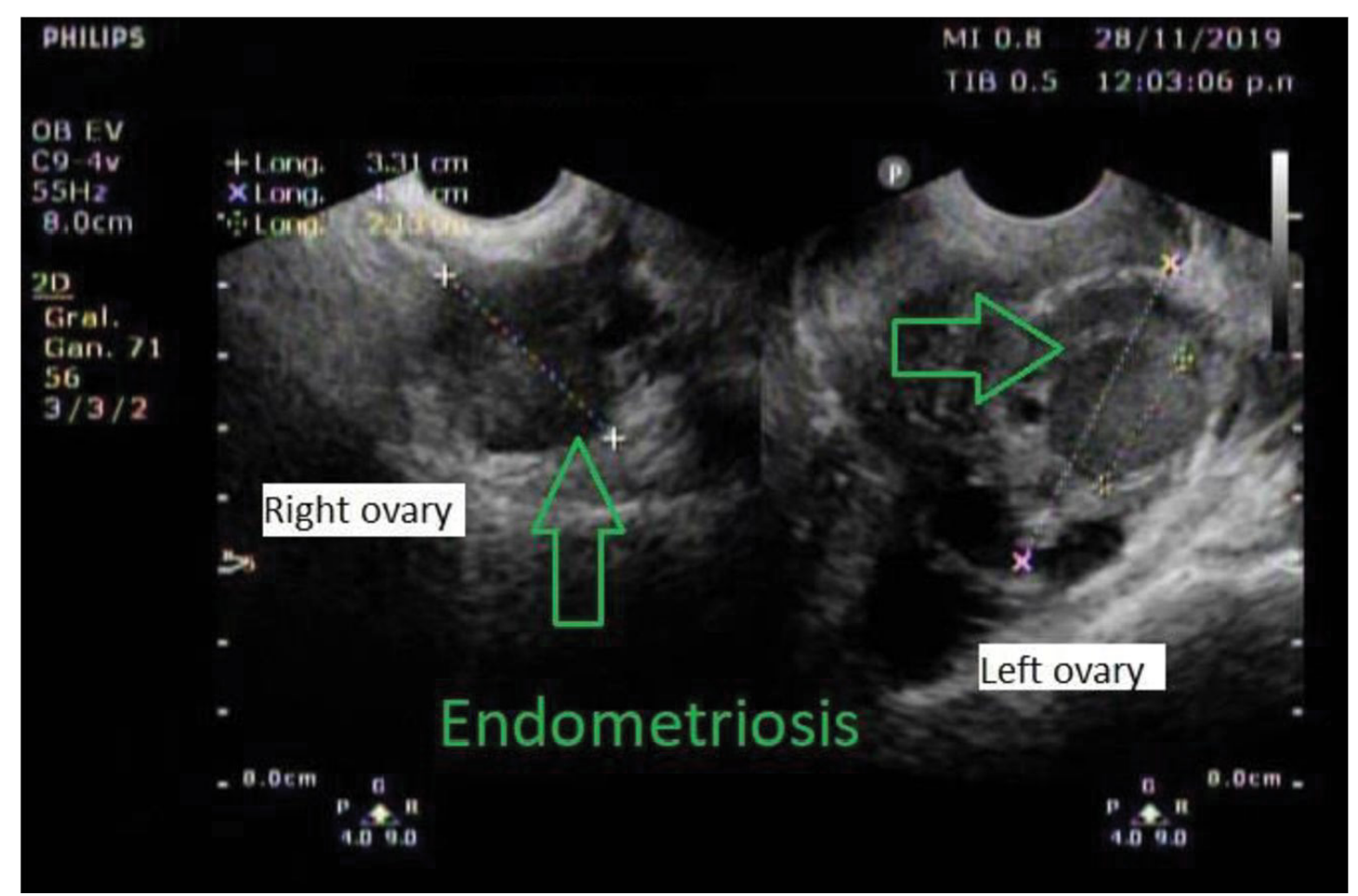

Figure 1: US findings: Right ovary within the normal size but with a complex solid mass. Left ovary with complex mass with a solid lesion measured $3 \mathrm{~cm}$. Doppler negative.

Copyright: (C) 2022 Leal C. This is an open-access article distributed under the terms of the Creative Commons Attribution License, which permits unrestricted use, distribution, and reproduction in any medium, provided the original author and source are credited. 


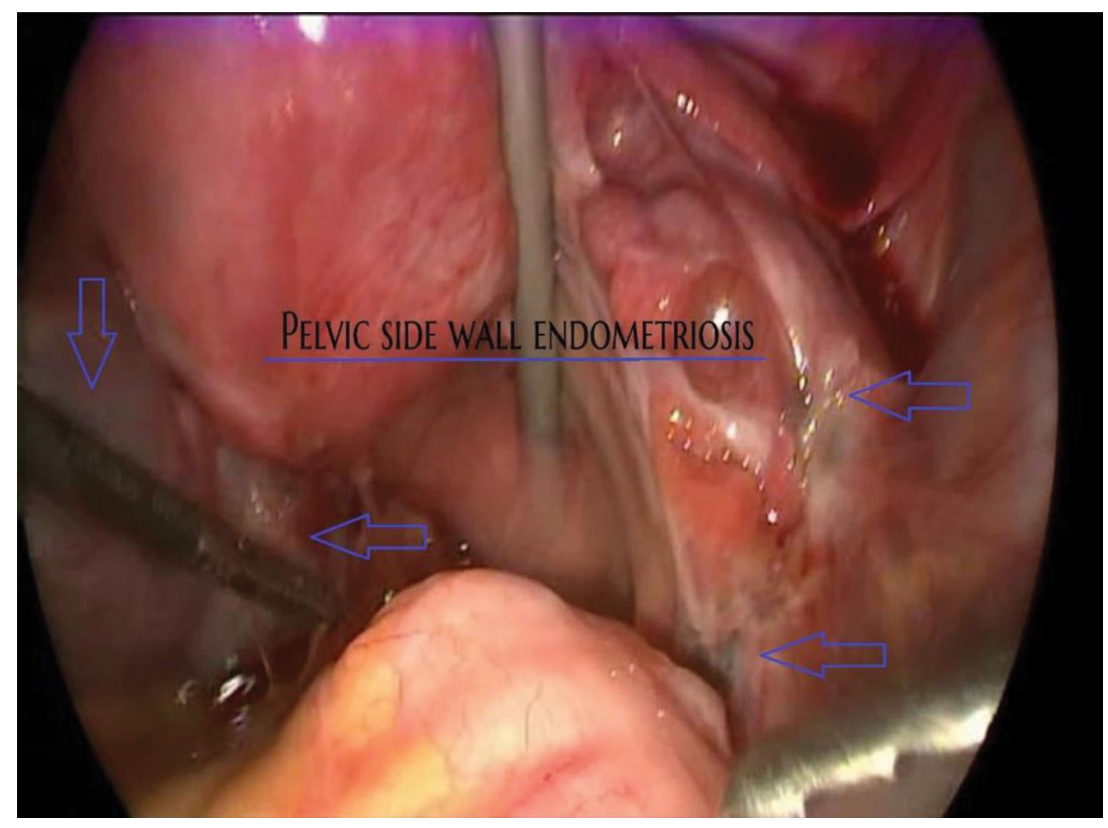

Figure 2: Initial laparoscopic inspection, showing evidence of infiltrative endometriosis, mainly on the right pelvic sidewall.

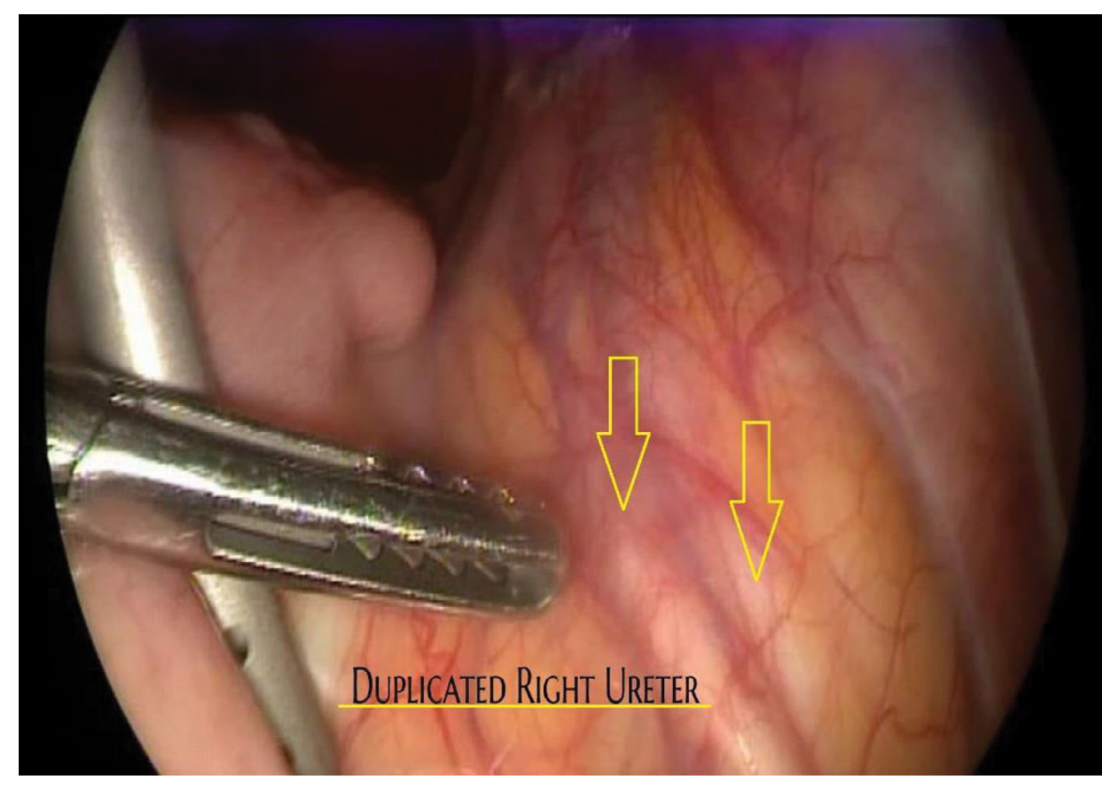

Figure 3: Laparoscopic view showing evidence of a duplex right ureter.

can have a normal insertion in the trigone, or it can be inserted ectopically in the bladder or elsewhere. Ectopic insertion of the ureter can result in obstruction or vesicoureteral reflux (VUR) [4]. Depending on the location of the ectopic insertion, incontinence also may be present.

Partial duplication is more common than complete duplication. In these cases, the kidney has two separate pelvicalyceal systems, with either a single ureter or two ureters that unite prior to insertion into the bladder. Strong knowledge of the retroperitoneal anatomy will facilitate the ureteral findings [5].

In patients with asymptomatic uncomplicated (no dilation)
*Corresponding author: Carlos Leal, MD, PhD, Division of Gynecologic Oncology, Department of Obstetrics and Gynecology, Universidad Autónoma de Chihuahua, Calle de La Llave \#1419 suite 103 Colonia Centro, CP 31000, Mexico, Tel: +52-(614)2353022

Accepted: January 29, 2022

Published online: January 31, 2022

Citation: Leal C (2022) "Duplex Ureter": ¿Are we Aware of this Uncommon Finding?. Ann Minim Invasive Surg 4(1):41-43 


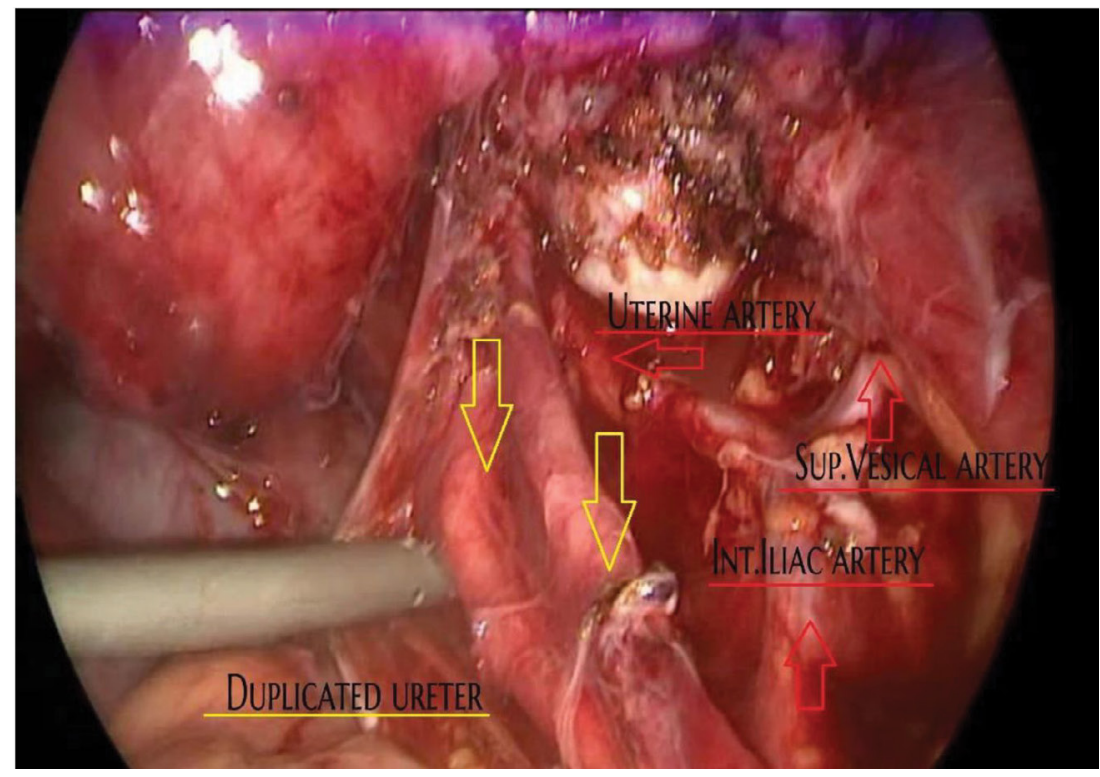

Figure 4: Retroperitoneal dissection of both right ureters; separating the retroperitoneal spaces and vessels anatomy.

duplication of the collecting system, no further intervention or referral is needed. However, if there is a history of urinary tract infection (UTI) or dilation (typically due to obstruction) further interventions must be done, this can be done with laparoscopic surgery.

\section{References}

1. Queisser-Luft A, Stolz G, Wiesel A, et al. (2002) Malformations in newborn: Results based on 30,940 infants and fetuses from the mainz congenital birth defect monitoring system (1990-1998). Arch Gynecol Obstet 266: 163-167.
2. Williams H (2007) Renal revision: From lobulation to duplication-what is normal? Arch Dis Child Educ Pract Ed 92: ep152-ep158.

3. Decter RM (1997) Renal duplication and fusion anomalies. Pediatr Clin North Am 44: 1323-1341.

4. Glassberg KI (2002) Normal and abnormal development of the kidney: A clinician's interpretation of current knowledge. J Urol 167: 2339-2350.

5. Carlos L, Espinoza V, Valenzuela A, et al. (2020) The Bermuda Triangle' a didactic description of the retroperitoneal anatomy. J Minim Invasive Gynecol 27: S118.

DOI: $10.36959 / 351 / 590$ 